\title{
Técnicas didácticas para la formación inicial de maestros. Programa "Enseñar como un campeón"
}

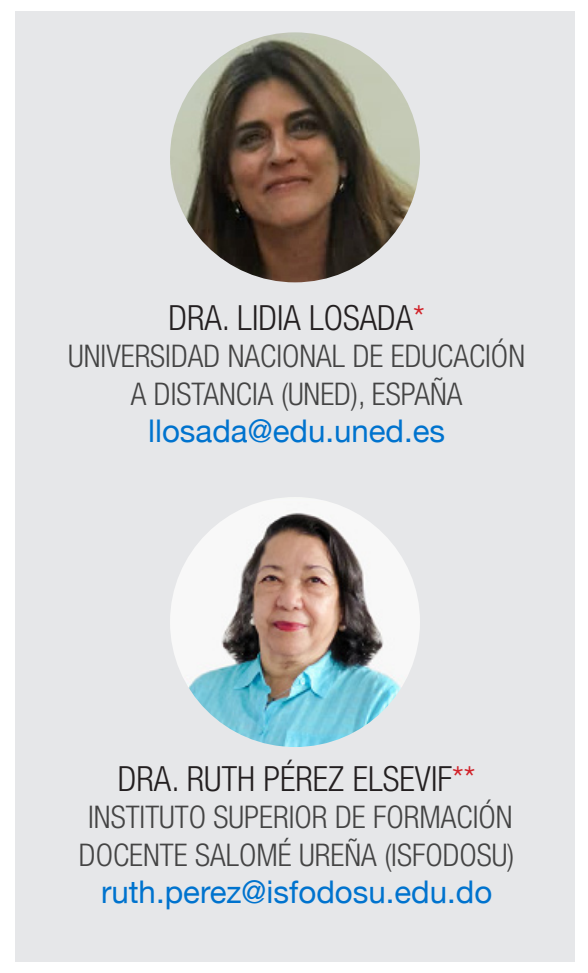

\author{
Teaching techniques for \\ initial teacher training: \\ "Teach like a champion" program
}

Recibido: 29 de octubre de 2019 | Aprobado: 28 de noviembre de 2019

\section{Resumen}

En el marco de la política del Gobierno Dominicano, los planteamientos tradicionales de la Educación Superior evolucionan hacia la formación de docentes de excelencia, respondiendo a la necesidad de hacer de los docentes agentes activos del desarrollo del país. La necesidad de cualificación del profesorado justifica actuaciones dirigidas a su formación, como el programa de entrenamiento "Enseñar como un campeón" (Lemov, 2010); una apropiada estrategia didáctica para la formación integral de los maestros. El propósito de este estudio consiste en proporcionar evidencias de los resultados cuantitativos y cualitativos de los efectos de esta intervención en los estudiantes de las licenciaturas de Educación, los cuales fueron entrenados para aplicar las técnicas en las escuelas. Se utilizó el método descriptivo para mostrar e interpretar el impacto de una realidad educativa tal como se manifestó y dar a conocer los resultados de la implementación de un programa académico. Para ello, se elaboró un instrumento ad hoc con el fin de estimar la autopercepción de los efectos del entrenamiento. Asimismo, se elaboró un cuestionario para la evaluación de la satisfacción de los estudiantes. Los resultados obtenidos evidencian la oportunidad de esta intervención, pues facilita la incorporación de competencias a la práctica educativa. Del análisis de los resultados se evidencia que la puesta en marcha de este proyecto ha sido una experiencia positiva para los estudiantes, así como para los docentes y puede servir de modelo para su implementación en el futuro.

Palabras clave: Didáctica; técnicas de enseñanza; formación docente; prácticas; innovación docente

\footnotetext{
* Doctora en Métodos de Investigación y Diagnóstico en Educación. Docente e Investigadora en la Facultad de Educación de la Universidad Nacional de Educación a Distancia (UNED) (España). Labora también para el Instituto Superior de Formación Docente Salomé Ureña (ISFODOSU). Para contactar a la autora: Ilosada@edu.uned.es

** Doctora en Liderazgo Educativo de Nova Southeastern University (NSU). Docente del Instituto Superior de Formación Docente Salomé Ureña (ISFODOSU). República Dominicana. Para contactar a la autora: ruth.perez@isfodosu.edu.do
} 


\section{Abstract}

In the framework of the Dominican Government's policy, the traditional approaches of Higher Education evolve towards the training of teachers of excellence, responding to the need of training active agents in the development of the country. The need for teacher qualification justifies actions aimed at their training, such as the training program "Teach as a champion" (Lemov, 2010); an appropriate didactic strategy for the integral formation of the teachers. The purpose of this study is to evaluate the effects of this intervention on the students of the Bachelor of Education. The students were trained to apply the techniques in the schools. An ad hoc instrument was used to estimate the effects of training. In addition, a questionnaire was prepared for the evaluation of student satisfaction. The results obtained show the opportunity of this intervention that facilitates the incorporation of competences into educational practice and can serve as a model for its implementation in the future.

Keywords: Didactic; teaching techniques; teacher training; practices; teaching innovation

\section{Introducción}

En el marco de la política del Gobierno Dominicano, los planteamientos tradicionales de la Educación Superior evolucionan hacia el enfoque de desarrollo de competencias para formar docentes de excelencia. La implementación de métodos y técnicas de enseñanza innovadores responde a la necesidad de hacer de los futuros docentes agentes activos de desarrollo del país a través de la educación. La formación de docentes de excelencia que contribuyan a mejorar la calidad del Sistema Educativo forma parte de la Meta Presidencial del Gobierno Dominicano. Las instituciones de Educación Superior del país, a través de la formación docente, están llamadas a llevar a cabo procesos educativos innovadores, que potencien el desarrollo de competencias humanas y profesionales.

Esta necesidad de cualificación del profesorado justifica actuaciones dirigidas a su formación, a través de programas de entrenamiento, tal es "Enseñar como un campeón" (Lemov, 2010), que se describe como una apropiada estrategia didáctica para la formación integral de los futuros maestros. "Enseñar como un campeón" consta de 49 técnicas didácticas que versan sobre el establecimiento de altas expectativas académicas, la planificación como medio de alcanzar el logro académico, la organización e impartición de las clases, la participación de los alumnos, la cultura del aula, el establecimiento y mantenimiento de altas expectativas de comportamiento, el desarrollo del carácter y la confianza, la creación de un ritmo positivo en el aula y el desarrollo del pensamiento crítico. En una segunda parte, el programa focaliza su atención en el desarrollo de las habilidades lectoras, incluyendo un material audiovisual que documenta la aplicación práctica de las técnicas.

El programa se posiciona al respecto de la disyuntiva de la enseñanza como arte o como ciencia susceptible de ser aprendida. Zabalza (2016) ha resuelto tal dilema, describiendo la enseñanza como una actividad compleja, en parte artística y en parte científica. Lemov (2010) postula que se puede aprender a enseñar y llegar a convertirse en un docente de excelencia a través del dominio de las técnicas, mediante su aprendizaje y práctica en el aula, restando importancia al talento innato esperable en un maestro.

Desde este planteamiento, asegurar la calidad de la formación docente implicaría un buen componente práctico junto a la supervisión de docentes experimentados. Además, este programa de entrenamiento resulta válido para ser aplicado en la actualización de los profesores en ejercicio y, quizás, en un futuro, el dominio de las técnicas pueda formar parte de los procesos de evaluación docente.

Lemov (2010) presenta estas técnicas eficaces de una manera práctica, sin adscribirse a paradigma educativo y como herramientas básicas que los buenos maestros aplican en el desarrollo de sus clases, consiguiendo niveles de rendimiento académico aceptables en los contextos más vulnerables. En la Tabla 1 se describen brevemente las técnicas descritas en el programa. 
Tabla 1. Síntesis de las técnicas del programa "Enseñar como un campeón" (Lemov, 2010)

\begin{tabular}{|c|c|c|}
\hline Dimensión & Técnica & Descripción \\
\hline \multirow{4}{*}{$\begin{array}{l}\text { 1. Establezca } \\
\text { altas expectativas } \\
\text { académicas }\end{array}$} & 1. Sin opción de salida. & $\begin{array}{l}\text { Todos deben participar y no se acepta responder "no sé". Llegado el momento, el } \\
\text { alumno renuente o incapaz de responder, deberá contestar correctamente cuando se le } \\
\text { pregunte, aunque solo sea para repetir la respuesta correcta. }\end{array}$ \\
\hline & $\begin{array}{l}\text { 2. Lo correcto es lo } \\
\text { correcto. }\end{array}$ & $\begin{array}{l}\text { Se debe exigir el } 100 \% \text {, sin aceptar respuestas parciales. Al insistir en la respuesta } \\
\text { correcta, el profesor hace ver que las preguntas y respuestas tienen importancia. Este } \\
\text { es un mensaje potente que el alumno recordará mucho tiempo después de haber dejado } \\
\text { el aula. }\end{array}$ \\
\hline & 8. Muéstrelo. & $\begin{array}{l}\text { Exhiba los objetivos trazados en un lugar visible de modo que los alumnos puedan com- } \\
\text { probar fácilmente el objeto de la clase del día. }\end{array}$ \\
\hline & 10. Plan doble. & $\begin{array}{l}\text { Además de lo que hará el profesor, en la planificación diaria mencione lo que los alumnos } \\
\text { deberán hacer a cada momento. Esto permite ver la clase desde la óptica de los alumnos } \\
\text { y mantenerlos participando de forma productiva. }\end{array}$ \\
\hline \multirow{3}{*}{$\begin{array}{l}\text { 3. Organice e imparta } \\
\text { las lecciones }\end{array}$} & 12. El gancho. & $\begin{array}{l}\text { Cuando sea necesario, haga una breve introducción orientada a entusiasmar a los } \\
\text { alumnos con el estudio de la materia. }\end{array}$ \\
\hline & 15. Camine por el salón. & Circule por el aula y haga responder y participar a los alumnos. \\
\hline & 16. Desglose. & $\begin{array}{l}\text { Si el curso no tiene claridad, responda desglosando el problema en sus elementos } \\
\text { constitutivos. }\end{array}$ \\
\hline \multirow{2}{*}{$\begin{array}{l}\text { 4. Haga participar a } \\
\text { sus alumnos en la } \\
\text { lección }\end{array}$} & $\begin{array}{l}\text { 22. Participación } \\
\text { imprevista. }\end{array}$ & $\begin{array}{l}\text { Interpele a todos los alumnos, levanten la mano o no. Deje en claro que todos tienen que } \\
\text { contestar preguntas, se hayan ofrecido o no. }\end{array}$ \\
\hline & 23. Llamar y responder. & $\begin{array}{l}\text { Haga una pregunta y pida a todo el curso contestar al unísono. Construya en el aula una } \\
\text { cultura de participación dinámica y positiva. }\end{array}$ \\
\hline \multirow{2}{*}{$\begin{array}{l}\text { 5. Cree una sólida } \\
\text { cultura del salón de } \\
\text { clases }\end{array}$} & 28. Rutina de entrada. & $\begin{array}{l}\text { Establezca una rutina eficaz, productiva y respetuosa respecto de la forma en que los } \\
\text { alumnos entran al aula, entregan sus deberes, toman asiento y se informan de lo que } \\
\text { harán ese día. }\end{array}$ \\
\hline & 29. Háganlo ahora. & $\begin{array}{l}\text { Dicte } 0 \text { anote en la pizarra, una breve actividad a realizar en el instante de entrar a } \\
\text { clases. La actividad debe ser un adelanto de la clase del día, no precisa explicación del } \\
\text { maestro ni discusión entre los alumnos, y requiere comprensión escrita. }\end{array}$ \\
\hline \multirow{3}{*}{$\begin{array}{l}\text { 6. Establezca y } \\
\text { mantenga altas } \\
\text { expectativas de } \\
\text { comportamiento }\end{array}$} & 36. 100 por ciento. & $\begin{array}{l}\text { Exija a cada alumno, sin excepción, el cumplimiento de las más altas normas de } \\
\text { conducta. Tres principios básicos: 1) intervenga de la forma menos invasiva posible; 2) } \\
\text { sea ponderado, firme y sereno; y 3) privilegie el buen comportamiento que usted pueda } \\
\text { comprobar. }\end{array}$ \\
\hline & 37. Qué hacer. & $\begin{array}{l}\text { Las instrucciones sobre conducta deben ser específicas, concretas, consecutivas y } \\
\text { observables. }\end{array}$ \\
\hline & 38. Voz fuerte. & $\begin{array}{l}\text { Los maestros transmiten autoridad en base a cinco principios básicos: 1) economía de } \\
\text { lenguaje: pocas palabras valen más que muchas;2) no trate de hablar al mismo tiempo: } \\
\text { espere a que guarden silencio; 3) no se salga del tema: evite discutir otras materias } \\
\text { mientras no se haya resuelto el tema que planteó; 4) mire de frente y con aplomo: párese } \\
\text { de frente al curso y no se mueva mientras habla; y 5) autoridad tranquila: cuando quiera } \\
\text { controlar al curso, hable pausado y más bajo que lo normal. }\end{array}$ \\
\hline \multirow{3}{*}{$\begin{array}{l}\text { 7. Desarrolle carácter } \\
\text { y confianza }\end{array}$} & 43. Encuadre positivo. & $\begin{array}{l}\text { Corrija de forma coherente y positiva; sitúe sus observaciones en el marco general del } \\
\text { mundo que quiere hacer ver a los alumnos. Concéntrese en lo que son capaces de hacer } \\
\text { ahora, espere siempre lo mejor y tenga una narrativa positiva. Conecte la conducta del } \\
\text { alumno con sus aspiraciones. }\end{array}$ \\
\hline & 44. Elogio preciso. & $\begin{array}{l}\text { Reconozca las conductas fuera de lo común y no simplemente el deber cumplido. Se } \\
\text { deben elogiar conductas que el alumno pueda controlar, no los rasgos o características } \\
\text { innatas. El elogio debe además ser sincero y hacerse en voz alta (y la crítica en voz baja). }\end{array}$ \\
\hline & 49. Norm & $\begin{array}{l}\text { Haga ver a los alumnos que, a nivel escolar, las respuestas incorrectas son normales y } \\
\text { tan importantes como las correctas. }\end{array}$ \\
\hline
\end{tabular}

Fuente: elaboración propia a partir de la propuesta de Puryear (2014). 
El programa "Enseñar como un campeón" se inserta en el sistema de prácticas docentes de una institución superior de formación docente dominicana, que se ha diseñado bajo el supuesto fundamental de articular la teoría con la práctica en contextos reales (ISFODOSU, 2018). Este sistema tiene una estructura que lo diferencia de otros programas nacionales de formación inicial de futuros docentes. En primer lugar, los estudiantes inician su práctica desde el primer año de carrera y desarrollan sus prácticas en las escuelas hasta el último año, en el que se insertan en un grado del ciclo y nivel que corresponde con el título al que optaron y durante un año escolar completo. En cada uno de los seis tramos de práctica docente los estudiantes desarrollan las competencias que se han definido en el perfil de egreso. En definitiva, las seis asignaturas o tramos de las Prácticas Docentes se constituyen como el hábitat ideal para el desarrollo de competencias de los futuros docentes, así como para evidenciar la validez y la confiabilidad de los instrumentos de observación del desempeño de los estudiantes durante su periodo de colaboración en los centros educativos.

La estrategia de acompañamiento que caracteriza este sistema de prácticas facilita la evaluación del programa aplicado a los fines de estudiar sus efectos, puesto que la estructura operativa del sistema está integrada por varios agentes significativos, como es el maestro anfitrión y el tutor acompañante, que han sido informantes clave para observar los efectos del programa. Gracias a su implicación, hemos podido estudiar el programa desde su entrenamiento a los estudiantes hasta su aplicación en las escuelas con los estudiantes de primaria y secundaria.

El programa "enseñar como un campeón" responde al objetivo de desarrollar competencias en los estudiantes, de acuerdo a los parámetros establecidos por la Normativa 9-2015 (MESCyT, 2015). El fin último es que los estudiantes sean capaces de aplicar las técnicas didácticas aprendidas en contextos reales, de manera que la estrategia de entrenamiento se complete con los aprendizajes propios de los paradigmas del aprendizaje situado (Brown, Collins y Duguid, 1989) y del aprendizaje experiencial (Kolb, 1984).

El objetivo de este estudio consiste en proporcionar evidencias de los resultados cuantitativos y cualitativos de los efectos de esta intervención en los estudiantes de las licenciaturas de Educación, los cuales fueron entrenados para aplicar las técnicas en las escuelas. A continuación, detallaremos la metodología empleada y el universo de participantes, también enumeramos las técnicas entrenadas y puestas en práctica y la descripción del instrumento. Más adelante, ofrecemos los resultados de autopercepción de los estudiantes participantes en el programa, estableciendo una comparación entre el grupo de Primaria y el grupo de Secundaria. Por último, ofrecemos unas conclusiones con una sucinta prospectiva que apunta a la ampliación del estudio.

\section{Método}

Para este estudio se utilizó un método descriptivo para mostrar e interpretar el impacto de una realidad educativa tal como se manifestó y dar a conocer los resultados de la implementación de un programa académico. El grupo de participantes estuvo compuesto por las licenciaturas de Educación Primaria $(n=20)$ y Licenciatura en Matemáticas Orientada a la Educación Secundaria $(n=24)$. La muestra fue de tipo incidental o por accesibilidad y se conforma, en su mayoría, por mujeres (70\%). La edad de los estudiantes abarca desde los 18 a los 27 años. Una docente, a su vez participante del equipo investigador de este estudio, impartió el programa dentro de la asignatura "Prácticas Docentes II", durante 16 sesiones de 2 horas de duración presenciales cada una. Una vez que los estudiantes aprendieron una serie de técnicas eficaces de enseñanza, contando con el acompañamiento del docente tutor, aplicaron las mismas en el período de prácticas en los centros educativos, durante 96 horas, en 12 semanas.

Se obtuvo la colaboración de los maestros de las escuelas que reciben estudiantes de prácticas de la institución. Durante el desarrollo de las intervenciones, el docente tutor llevó a cabo la observación de la aplicación de las técnicas entrenadas previamente, junto con los maestros anfitriones de las escuelas en las que los estudiantes desarrollan sus prácticas. Los estudiantes estuvieron informados del propósito del estudio; 
se les solicitó su participación voluntaria y se les informó del carácter anónimo y confidencial de los resultados.

Se utilizó un instrumento elaborado ad hoc con el fin de estimar la opinión de los evaluadores sobre los efectos del entrenamiento de las técnicas y la efectividad en su aplicación en el aula. Asimismo, se elaboró un cuestionario para la evaluación de la satisfacción de los estudiantes con los aprendizajes logrados.
A continuación, se presentan las técnicas entrenadas y puestas en práctica, que pueden ser revisadas en el texto original de Lemov (2010), de fácil acceso a través de Google Scholar. En un principio se había pensado en seguir el orden de dimensiones a trabajar de acuerdo a las que ofrece este documento base que referimos, pero consideramos conveniente profundizar en una selección, en lugar de pasear por todas. Los criterios para escoger las técnicas fueron novedad y disponibilidad de tiempo.

Tabla 2. Técnicas entrenadas y puestas en práctica

\begin{tabular}{|c|c|c|}
\hline Dimensión & Descripción & Técnicas \\
\hline Dimensión 1 & $\begin{array}{l}\text { Establezca altas expectativas } \\
\text { académicas }\end{array}$ & $\begin{array}{l}\text { Técnica 1. Sin opción de salida } \\
\text { Técnica 2. Lo correcto es lo correcto } \\
\text { Técnica 3. Extiéndalo }\end{array}$ \\
\hline Dimensión 2 & $\begin{array}{l}\text { Haga su planeación para asegurar el } \\
\text { logro académico }\end{array}$ & Técnica 8. Muéstrelo \\
\hline Dimensión 3 & Organice e imparta las lecciones & $\begin{array}{l}\text { Técnica 12. El gancho } \\
\text { Técnica 14. Pizarrón y Papel } \\
\text { Técnica 15. Camine por el salón } \\
\text { Técnica 16. Desglose } \\
\text { Técnica 20. Boleto de salida }\end{array}$ \\
\hline Dimensión 4 & $\begin{array}{l}\text { Haga participar a sus alumnos en la } \\
\text { lección }\end{array}$ & $\begin{array}{l}\text { Técnica 22. Participación imprevista } \\
\text { Técnica 24. Pepper game } \\
\text { Técnica 27. Vegas }\end{array}$ \\
\hline Dimensión 6 & $\begin{array}{l}\text { Establezca y mantenga altas } \\
\text { expectativas de comportamiento }\end{array}$ & $\begin{array}{l}\text { Técnica 36. } 100 \text { por ciento } \\
\text { Técnica 37. Qué hacer } \\
\text { Técnica 38. Voz fuerte } \\
\text { Técnica 39. Háganlo otra vez }\end{array}$ \\
\hline Dimensión 7 & Desarrolle carácter y confianza & $\begin{array}{l}\text { Técnica 43. Encuadre positivo } \\
\text { Técnica 44. Elogio preciso } \\
\text { Técnica 45. Afectuoso y estricto } \\
\text { Técnica 49. Normalice el error }\end{array}$ \\
\hline Dimensión 8 & $\begin{array}{l}\text { Mejore su ritmo. } \\
\text { a) Cambie el ritmo } \\
\text { b) Todas las manos } \\
\text { c) Cada minuto cuenta }\end{array}$ & \\
\hline Dimensión 9 & $\begin{array}{l}\text { Desafíe a los alumnos a pensar de } \\
\text { manera crítica: } \\
\text { a) Una a la vez } \\
\text { b) De lo simple a lo complejo }\end{array}$ & \\
\hline
\end{tabular}




\section{Instrumentos}

Cuestionario de evaluación de los estudiantes. Consta de ocho ítems construidos ad hoc para cada una de las técnicas entrenadas. Se solicita que los evaluadores respondan sobre una escala de cuatro grados: 1) insatisfactorio, 2) básico, 3) avanzado y 4) sobresaliente. Los estudiantes son evaluados por el docente tutor de la asignatura, así como por el maestro anfitrión. Para cada una de las técnicas se elaboraron ocho ítems específicos, buscando la coherencia y significatividad para esa técnica en concreto. Por ejemplo, para la técnica "Trabajan con el reloj; cada minuto cuenta", los ocho ítems fueron: 1) la participación en la clase permitió el aprendizaje; 2) fue fácil poner en práctica la técnica utilizada; 3) hubo respeto durante el proceso en el estudiantado; 4) la técnica ejecutada permitió el aprendizaje significativo; 5) se percibió que no hubo pérdida del tiempo; 6) siempre se puede enseñar con esta técnica; 7) se evidenció durante la clase el manejo de reloj; 8) se hizo hincapié en la importancia de cada segundo. En el Anexo 1 se muestra la estructura del instrumento, en este caso para la evaluación de la técnica: "Sin Opción de Salida".

Cuestionario de autoevaluación sobre la satisfacción en la participación del programa de entrenamiento en técnicas de enseñanza (Anexo 2). Las instrucciones de este cuestionario informan al estudiante sobre el propósito de evaluar su satisfacción como participante en el programa de entrenamiento en técnicas didácticas vinculadas al proyecto "Enseñar como un campeón"; se solicita que responda con absoluta sinceridad a cada una de las afirmaciones sobre las técnicas aprendidas y su posterior aplicación durante su inserción en la escuela, como estudiante en prácticas. El cuestionario consta de 16 ítems que se distribuyen en tres secciones relativas a la percepción de los estudiantes sobre las técnicas. En la primera sección el estudiante responde a los ocho ítems en una escala conformada por "no aplica"; "pocas veces"; "con frecuencia"; "siempre": 1) promueve el clima positivo; 2) favorece la interacción entre docente y estudiante; 3) permite detectar necesidades individuales del estudiante: sus intereses, sus motivaciones y capacidad para aprender; 4) favorece el manejo de la conducta en el aula; 5) permite una distribución del tiempo efectiva; 6) promueve el aprendizaje significativo; 7) mejora la calidad de la retroalimentación que se ofrece al estudiante; 8) favorece la atención de los estudiantes.

La segunda sección tiene una escala de respuesta de tres niveles "poco"; "bastante" y "mucho"; está conformada por siete ítems: 1) me interesó participar en el proyecto; 2) al leer sobre las técnicas, sentí curiosidad por aprender; 3) me ha parecido que estas técnicas son importantes para mi futuro profesional; 4) he tenido inconvenientes para aplicar las técnicas; 5) he sido capaz de integrar más de una técnica durante la inmersión en la escuela; 6) estoy satisfecho con el aprendizaje de estas técnicas y 7) pienso que utilizaré estas técnicas en el futuro.

En la tercera sección, conformada por una única pregunta, se solicita a los estudiantes que valoren su nivel de dominio en cada una de las técnicas, con las siguientes opciones de respuesta: "no aplica";" insuficiente"; "básico"; "avanzado". Finalmente, el cuestionario ofrece un espacio para incorporar las observaciones que el estudiante crea oportunas.

\section{Resultados}

Los resultados obtenidos se presentan a continuación en sus dos vertientes: 1) la evaluación de los estudiantes por parte de los docentes (docente de la asignatura o Maestro tutor, más el maestro anfitrión de la escuela) y 2) la satisfacción de los estudiantes sobre las técnicas entrenadas, practicadas y objeto de observación.

Resultados de la evaluación de los docentes a los estudiantes.

Los docentes evaluadores calificaron a los estudiantes en cada una de las técnicas entrenadas en los niveles 2) básico, 3) avanzado y 4) sobresaliente. Salvo escasas excepciones, el mayor porcentaje de respuesta se sitúa en el nivel 3) y 4) a lo largo de los diversos ítems de los que consta cada técnica. No se registraron respuestas en el nivel 1) insatisfactorio. Esta tendencia ocurre tanto para la evaluación realizada por el Maestro tutor de la asignatura de prácticas, como para la evaluación realizada por el Maestro anfitrión de la escuela. 
Resultados de autopercepción de los estudiantes sobre las técnicas objeto de observación que fueron entrenadas y practicadas.

Los resultados relativos a la autopercepción se presentan en las Tablas 3, 4 y 5, en las cuales se puede observar la frecuencia y porcentaje en cada uno de los ítems del instrumento aplicado a los estudiantes, tanto para el grupo de la Licenciatura en Educación Primaria, como para el grupo de la Licenciatura en Educación Secundaria.
Así, se hallaron los siguientes resultados para la Sección I del cuestionario, con una escala de respuesta: "no aplica"; "pocas veces"; "con frecuencia"; "siempre". El porcentaje total se ha calculado para cada ítem y corresponde a la suma de respuestas en la escala, dividido entre el total de respuestas en todas las escalas.

1. Promueve el clima positivo: el $64.71 \%$ del grupo de Educación Primaria manifestó que se promovió con frecuencia, mientras que $61.54 \%$ del grupo de Secundaria afirmó que

Tabla 3. Resultados de la autopercepción de los estudiantes. Sección I

\begin{tabular}{|c|c|c|c|c|c|c|c|c|c|c|c|c|}
\hline \multirow{3}{*}{ VARIABLES SECCIÓN I } & \multicolumn{6}{|c|}{ Grupo Primaria } & \multicolumn{6}{|c|}{ Grupo Secundaria } \\
\hline & \multicolumn{2}{|c|}{ Siempre } & \multicolumn{2}{|c|}{$\begin{array}{l}\text { Con } \\
\text { frecuencia }\end{array}$} & \multicolumn{2}{|c|}{$\begin{array}{l}\text { Pocas } \\
\text { veces }\end{array}$} & \multicolumn{2}{|c|}{ Siempre } & \multicolumn{2}{|c|}{$\begin{array}{c}\text { Con } \\
\text { frecuencia }\end{array}$} & \multicolumn{2}{|c|}{$\begin{array}{l}\text { Pocas } \\
\text { veces }\end{array}$} \\
\hline & $\mathrm{F}$ & $\%$ & $\mathrm{~F}$ & $\%$ & $\mathrm{~F}$ & $\%$ & $\mathrm{~F}$ & $\%$ & $\mathrm{~F}$ & $\%$ & $\mathrm{~F}$ & $\%$ \\
\hline Promueve el clima positivo & 6 & 35.29 & 11 & 64.71 & 0 & 0.00 & 8 & 61.54 & 4 & 30.77 & 1 & 7.69 \\
\hline $\begin{array}{l}\text { Favorece la interacción entre } \\
\text { docente y estudiante }\end{array}$ & 10 & 58.82 & 5 & 29.42 & 2 & 11.76 & 8 & 61.54 & 4 & 30.77 & 1 & 7.69 \\
\hline $\begin{array}{l}\text { Permite detectar necesidades del } \\
\text { estudiante: intereses, motivaciones } \\
\text { y capacidad de aprender }\end{array}$ & 9 & 52.94 & 7 & 41.18 & 1 & 5.88 & 8 & 61.54 & 4 & 30.77 & 1 & 7.69 \\
\hline $\begin{array}{l}\text { Favorece el manejo de la conducta } \\
\text { en el aula }\end{array}$ & 7 & 41.18 & 9 & 52.94 & 1 & 5.88 & 8 & 61.54 & 4 & 30.77 & 1 & 7.69 \\
\hline $\begin{array}{l}\text { Permite una distribución efectiva } \\
\text { del tiempo }\end{array}$ & 4 & 23.53 & 7 & 41.18 & 6 & 35.29 & 8 & 61.54 & 4 & 30.77 & 1 & 7.69 \\
\hline $\begin{array}{l}\text { Promueve el aprendizaje } \\
\text { significativo }\end{array}$ & 4 & 23.53 & 7 & 41.18 & 6 & 35.29 & 8 & 61.54 & 4 & 30.77 & 1 & 7.69 \\
\hline $\begin{array}{l}\text { Mejora la calidad de la } \\
\text { retroalimentación ofrecida }\end{array}$ & 5 & 29.41 & 9 & 22.94 & 3 & 17.64 & 8 & 61.54 & 4 & 30.77 & 1 & 7.69 \\
\hline $\begin{array}{l}\text { Favorece la atención de los } \\
\text { estudiantes }\end{array}$ & 12 & 70.59 & 4 & 23.53 & 1 & 5.88 & 8 & 61.54 & 4 & 30.77 & 1 & 7.69 \\
\hline
\end{tabular}


se promovió siempre. En total, un 50.00\% respondió que con frecuencia y un $46.67 \%$ respondió que siempre.

2. Favorece la interacción entre docente y estudiante: el 58.82\% del grupo de Educación Primaria y el $61.54 \%$ del grupo de Secundaria, afirmaron que siempre favoreció. En total, el $60 \%$ afirmó que siempre favoreció esta interacción.

3. Permite detectar necesidades individuales del estudiante: sus intereses, sus motivaciones y capacidad para aprender: afirmaron que siempre permitió detectar estas necesidades individuales de los estudiantes; el 52.94\% del grupo de Educación Primaria y el 61.54\% del grupo de Secundaria.

4. Favorece el manejo de la conducta en el aula: el 52.94\% del grupo de Primaria consideró que, con frecuencia, se favoreció el manejo de la conducta en el aula; el 61.54\% del grupo de Secundaria afirmó que siempre se favorece; $50.00 \%$, si se consideran ambos grupos.

5. Permite una distribución efectiva del tiempo: tanto el $41.18 \%$ del grupo de Primaria como el $61.54 \%$ del grupo de Secundaria consideraron que siempre permitió una distribución efectiva del tiempo. El 40\% del total afirmó que siempre se promovió.

6. Promueve el aprendizaje significativo: el 41.18\% del grupo de Primaria consideró que se promovió con frecuencia; el $61.54 \%$ del grupo de Secundaria consideró que siempre se promovió. El 40\% del total afirmó que siempre se promovió.

7. Mejora la calidad de la retroalimentación que se ofrece al estudiante: el 52.94\% del grupo de Primaria respondió que se hace con frecuencia; el 61.54\% del grupo de Secundaria seleccionó siempre. En total, el 43.33\% opinó que con frecuencia y otro $43.33 \%$ afirmó que siempre.

8. Favorece la atención de los estudiantes: Para el $70.59 \%$ del Grupo Primaria y para el $61.54 \%$ del Grupo de Secundaria, siempre favoreció la atención de los estudiantes.

Para la segunda sección se hallaron los siguientes resultados de autopercepción de los estudiantes, a lo largo de los siete ítems y según la escala de respuesta "poco"; "bastante" y "mucho". El porcentaje total se ha calculado para cada ítem y

Tabla 4. Resultados de la autopercepción de los estudiantes. Sección II

\begin{tabular}{|c|c|c|c|c|c|c|c|c|c|c|c|c|}
\hline \multirow{3}{*}{ VARIABLES SECCIÓN II } & \multicolumn{6}{|c|}{ Grupo Primaria } & \multicolumn{6}{|c|}{ Grupo Secundaria } \\
\hline & \multicolumn{2}{|c|}{ Mucho } & \multicolumn{2}{|c|}{ Bastante } & \multicolumn{2}{|c|}{ Poco } & \multicolumn{2}{|c|}{ Mucho } & \multicolumn{2}{|c|}{ Bastante } & \multicolumn{2}{|c|}{ Poco } \\
\hline & $\mathrm{F}$ & $\%$ & $\mathrm{~F}$ & $\%$ & $\mathrm{~F}$ & $\%$ & $\mathrm{~F}$ & $\%$ & $\mathrm{~F}$ & $\%$ & $\mathrm{~F}$ & $\%$ \\
\hline Me interesó participar en el proyecto & 10 & 58.82 & 7 & 41.18 & 0 & 0.00 & 10 & 76.92 & 3 & 23.08 & 0 & 0.00 \\
\hline $\begin{array}{c}\text { Al leer sobre las técnicas sentí curiosidad por } \\
\text { aprender }\end{array}$ & 7 & 41.18 & 9 & 52.94 & 1 & 5.88 & 9 & 69.23 & 3 & 23.08 & 1 & 7.69 \\
\hline $\begin{array}{l}\text { Me ha parecido que estas técnicas son importantes } \\
\text { para mi futuro profesional. }\end{array}$ & 10 & 58.82 & 7 & 41.18 & 0 & 0.00 & 11 & 84.62 & 1 & 7.69 & 1 & 7.69 \\
\hline He tenido inconvenientes para aplicar las técnicas & 1 & 5.88 & 3 & 17.65 & 13 & 76.47 & 0 & 0.00 & 1 & 7.69 & 12 & 92.31 \\
\hline $\begin{array}{l}\text { He sido capaz de integrar más de una técnica } \\
\text { durante la inmersión en la escuela }\end{array}$ & 9 & 52.94 & 6 & 35.29 & 2 & 11.76 & 5 & 38.46 & 5 & 38.46 & 3 & 23.08 \\
\hline Estoy satisfecho con el aprendizaje de las técnicas & 9 & 52.94 & 8 & 47.06 & 0 & 0.00 & 10 & 76.92 & 3 & 23.08 & 0 & 0.00 \\
\hline Pienso que utilizaré estas técnicas en el futuro & 13 & 76.47 & 4 & 23.53 & 0 & 0.00 & 12 & 92.31 & 1 & 7.69 & 0 & 0.00 \\
\hline
\end{tabular}


corresponde a la suma de respuestas en la escala, dividido entre el total de respuestas en todas las escalas.

1. Me interesó participar en el proyecto: el $66.67 \%$ de ambos grupos se interesó mucho en participar; el 58.82\% y el 76.92\%, en Primaria y Secundaria, respectivamente.

2. Al leer sobre las técnicas, sentí curiosidad por aprender: el grupo de Primaria (52.94\%) sintió bastante curiosidad por aprender; el 69.23\% del grupo de Secundaria sintió mucha curiosidad. El 53.33\% de ambos grupos manifestó sentir mucha curiosidad.

3. Me ha parecido que estas técnicas son importantes para mi futuro profesional: el grupo de Primaria (58.82\%) y el grupo de Secundaria (84.62\%) consideraron que estás técnicas tienen mucha importancia. En total, el 70\% consideró que estas técnicas son bastante importantes.

4. He tenido inconvenientes para aplicar las técnicas: el grupo de Primaria (76.47\%) y el grupo de Secundaria (92.31\%), contestaron que han tenido pocos inconvenientes para su aplicación. En total, un 83.33\% de los miembros de ambos grupos consideró que ha habido pocos inconvenientes para la aplicación de estas técnicas.

5. He sido capaz de integrar más de una técnica durante la inmersión en la escuela: el grupo de Primaria (52.94\%) manifestó que mucho, mientras que un $38.46 \%$ del grupo de Secundaria se posicionó en "mucho" y otro $38.46 \%$ en "bastante".

6. Estoy satisfecho con el aprendizaje de estas técnicas: el grupo de Primaria (52.94\%) y el grupo de Secundaria (76.92\%) se posicionaron en "mucho". En total, el 63.33\% está muy satisfecho y el restante $36.67 \%$ está bastante satisfecho al haber aprendido estas técnicas.

7. Pienso que utilizaré estas técnicas en el futuro: el $76.47 \%$ del grupo de Primaria y el $92.31 \%$ del grupo de Secundaria declararon que utilizarán mucho estas técnicas en el futuro. Considerando las respuestas de ambos grupos, el $84.33 \%$ afirmó que las utilizará mucho en el futuro.

Tabla 5. Resultados de la autopercepción de los estudiantes. Sección III

\begin{tabular}{|c|c|c|c|c|c|c|c|c|c|c|c|c|}
\hline \multirow{3}{*}{ VARIABLE SECCIÓN III } & \multicolumn{6}{|c|}{ Grupo Primaria } & \multicolumn{6}{|c|}{ Grupo Secundaria } \\
\hline & \multicolumn{2}{|c|}{ Básico } & \multicolumn{2}{|c|}{ Avanzado } & \multicolumn{2}{|c|}{ Insuficiente } & \multicolumn{2}{|c|}{ Básico } & \multicolumn{2}{|c|}{ Avanzado } & \multicolumn{2}{|c|}{ Insuficiente } \\
\hline & $\mathrm{F}$ & $\%$ & $\mathrm{~F}$ & $\%$ & $\mathrm{~F}$ & $\%$ & $\mathrm{~F}$ & $\%$ & $\mathrm{~F}$ & $\%$ & $\mathrm{~F}$ & $\%$ \\
\hline $\begin{array}{l}\text { Considero que mi nivel de } \\
\text { dominio de esta técnica es }\end{array}$ & 6 & 35.29 & 11 & 64.71 & 0 & 0.00 & 10 & 76.92 & 3 & 23.08 & 0 & 0.00 \\
\hline
\end{tabular}

En la tercera sección del cuestionario, solo hay un ítem: considero que mi nivel de dominio en cada una de las técnicas es...; según la escala de respuesta, el $64.71 \%$ del grupo de Primaria seleccionó avanzado, mientras que el $76.92 \%$ del grupo de Secundaria se posicionó en un nivel de dominio básico. En general, el 53.33\% consideró que era básico.

Finalmente, el cuestionario de autopercepción ofrecía un espacio para incorporar opiniones sobre la valoración de las técnicas, de las cuáles se presenta una síntesis a continuación: 
Tabla 6: Opiniones sobre la valoración de las técnicas

\begin{tabular}{|c|c|c|}
\hline Dimensión & Técnica & Opiniones sobre la valoración de las técnicas \\
\hline \multirow{2}{*}{$\begin{array}{l}\text { Dimensión } 2 \text {. Haga su } \\
\text { planeación para asegurar el } \\
\text { logro académico. }\end{array}$} & Muéstrelo. & $\begin{array}{l}\text { "Colocando en un lugar visible del salón el objetivo todos los días, } \\
\text { sus alumnos y colegas se enteran y ayuda a los estudiantes a } \\
\text { mantener el tema presente, de esta forma al retroalimentar ellos } \\
\text { miran y fijan sus saberes". }\end{array}$ \\
\hline & El gancho. & $\begin{array}{l}\text { "Con esta técnica, no solo aprendieron los estudiantes, sino } \\
\text { también la participante. Mediante una introducción corta se } \\
\text { fomentó el interés y se logró el aprendizaje sobre la cultura } \\
\text { taína". }\end{array}$ \\
\hline $\begin{array}{l}\text { Dimensión 4. Haga participar a } \\
\text { sus alumnos en la lección. }\end{array}$ & Vegas. & $\begin{array}{l}\text { "En esta técnica, se implementa el juego, pero siempre y cuando } \\
\text { sea acorde al tema de enseñanza, porque de lo contrario, } \\
\text { distraería el objetivo de la clase y, a la vez, se limita para que el } \\
\text { juego no continúe a espalda del maestro". }\end{array}$ \\
\hline \multirow{4}{*}{$\begin{array}{l}\text { Dimensión 6. Establezca y } \\
\text { mantenga altas expectativas de } \\
\text { comportamiento. }\end{array}$} & 100 por ciento. & $\begin{array}{l}\text { "Con esta técnica, se realizan diferentes intervenciones } \\
\text { en el aula y se mantiene un ambiente de orden y disciplina; } \\
\text { efectivamente, atrae la atención de los niños". }\end{array}$ \\
\hline & ¿Qué hacer? & $\begin{array}{l}\text { "Esta técnica trata que los alumnos sepan qué deben hacer, con } \\
\text { orientaciones claras y precisas y motivando, para que cumplan } \\
\text { con su hacer en el aula". }\end{array}$ \\
\hline & Voz fuerte. & $\begin{array}{l}\text { "Hablar claro y fuerte permite que los estudiantes entiendan el } \\
\text { mandato; así perciben las iniciativas y seguridad del docente a } \\
\text { la hora de impartir las instrucciones". }\end{array}$ \\
\hline & Encuadre positivo. & $\begin{array}{l}\text { "Esta técnica ayudó a que los alumnos estuvieran activos y más } \\
\text { atentos a participar, pues la intervención siempre se trabaja en } \\
\text { positivo, de manera que entiendan que pueden lograrlo". }\end{array}$ \\
\hline \multirow[b]{2}{*}{ Dimensión 8. Mejore su ritmo. } & Todas las manos. & $\begin{array}{l}\text { "Esta técnica permitió impartir la clase organizada, observando } \\
\text { que, si relacionamos el tema con algo de su interés, los alumnos } \\
\text { prestan más atención, son más activos y, de manera clara, } \\
\text { entienden la explicación". }\end{array}$ \\
\hline & Cambie el ritmo. & $\begin{array}{l}\text { "Siendo esta mi primera clase y primera vez usando esta } \\
\text { técnica, me siento algo nerviosa; los estudiantes colaboraron } \\
\text { con las orientaciones y el tema trabajado -las operaciones } \\
\text { combinadas y la jerarquía-, fueron a la pizarra y realizaron las } \\
\text { operaciones indicadas". }\end{array}$ \\
\hline
\end{tabular}




\section{Conclusiones}

El presente trabajo proporciona evidencias relativas a los efectos de una intervención basada en un programa prestigioso en el entorno estadounidense, el cual ha sido adaptado al contexto dominicano y, más específicamente, a una institución de Educación Superior especializada en la formación de maestros. Los procesos llevados a cabo para la enseñanza y el aprendizaje de estas técnicas eficaces del programa "Enseñar como un campeón", han formado parte de la estrategia educativa de formar docentes de excelencia en el marco de la asignatura de prácticas docentes.

Lemov (2010) considera que estas habilidades de manejo del aula son fundamentales para el docente y eficaces para el aprendizaje de los alumnos, puesto que la óptima organización del aula, el establecimiento de rutinas, la generación de participación o la gestión eficaz del tiempo, se han mostrado como facilitadores del aprendizaje, así como lo son, también, la generación de confianza en sus alumnos y la puesta en marcha de instrucciones claras y con voz enérgica.

En cualquier caso, con la aplicación de programas de intervención como este para la enseñanza de tales técnicas efectivas, de manera intencional y sistematizada, se evidencia el papel de la educación como agente de cambio, a través del cual se puede modificar la conducta y el rendimiento de los estudiantes (Beltrán Llera, 2013).

Programas como "Enseñar como un campeón" cumplen con la función de formar docentes efectivos y proporcionar alta calidad en la enseñanza (Kretchmar y Zeichner, 2016), además de tener sus efectos en la educación inclusiva y la transformación social (Schlessinger y Oyler, 2015; Zeichner, 2016). Los resultados obtenidos permitieron identificar la eficacia al aplicar las técnicas de los estudiantes en prácticas, así como analizar la satisfacción de los estudiantes sobre las técnicas. En este sentido, las observaciones de los estudiantes funcionaron como complemento a la evaluación de los observadores y fomentaron la propia autoevaluación y reflexión de los estudiantes a lo largo de los indicadores, ya que en estas opiniones se aprecia la toma de conciencia de la importancia de utilizar las mejores estrategias para producir aprendizajes eficaces.
Del análisis de los resultados se evidencia que la puesta en marcha de este proyecto ha sido una experiencia positiva para los estudiantes, así como para los docentes.

Asimismo, en futuros estudios se pretende contar con una muestra mayor de discentes que afecte a la diversidad de titulaciones que oferta la institución, de manera que se puedan llevar a cabo análisis más avanzados y sea posible la generalización de los resultados, incorporando las conclusiones a las siguientes intervenciones. No obstante, aunque el alcance de su aplicación no fue demasiado ambicioso, se puede concluir que los resultados fueron satisfactorios en ambos tipos de evaluación y en todas las dimensiones y muestran la oportunidad de esta intervención, de manera que sirva de modelo para la implementación de este programa innovador en el futuro.

Aventuramos que el programa puede promover la mejora del desempeño futuro del docente, pues facilita el desarrollo de competencias imprescindibles para un maestro y consolida la vinculación de los aprendizajes teóricos con la experiencia práctica mediante la inserción en centros educativos que fomenten el aprendizaje situado y experiencial.

\section{Referencias bibliográficas}

Beltrán Llera, J. A. (2013). La educación como cambio. Revista Española de Pedagogía. 71(274), 101-118. ISSN: 0064-9461 (impreso); 2174-0909 (Online) España. Recuperado desde: https://revistadepedagogia.org/lxxi/no-254/laeducacion-como-cambio/101400010298/

Brown, J. S., Collins, A. y Duguid, P. (1989). Situated cognition and the culture of learning. Educational Researcher, 18(1), 32-45.

ISFODOSU (2018). Sistema de Prácticas Docentes del ISFODOSU. Serie Prácticas Docentes 1. ISFODOSU. Santo Domingo: República Dominicana.

Lemov, D. (2010). Enseña como un campeón: 49 técnicas de enseñanza para colocar a tus alumnos en la ruta del éxito (Teach like a champion: 49 techniques that put students on the path to college). México: Limusa Wiley. 
Ministerio de Educación Superior Ciencia y Tecnología (MESCYT) (2015). Normativa 9-2015 para la Formación Docente de Calidad en la República Dominicana. Santo Domingo: República Dominicana.

Puryear, J. (2014). ¿Quéhacen los grandes maestros? Las técnicas que separan a los sobresalientes del promedio. Resumen de "Enseña como un Campeón" del autor Doug Lemov. Revista Inter-American Dialogue. Mayo 2014. Estados Unidos de América: Organización de los Estados Americanos [OEA]. Recuperado desde: http:// recursos. portaleducoas.org/publicaciones/quhacen-los-grandes-maestros-las-t-cnicas-queseparan-los-sobresalientes-del-promedio

Kolb, D.A. (1984). Experiential learning: Experience as the source of learning and development. Englewood Cliffs, NJ: Prentice-Hall.

Kretchmar, K., y Zeichner, K. (2016). Teacher prep 3.0: A vision for teacher education to impact social transformation. Journal of Education for Teaching, 42(4), 417-433. Seattle, Washington: Estados Unidos de Norteamérica. Recuperado desde: https://www.researchgate.net/ publication/307590184_Teacher_prep_30_a_ vision_for_teacher_education_to_impact_ social_transformation

Schlessinger, S., y Oyler, C. (2015). Commentary: Inquiry-Based Teacher Learning for Inclusivity: Professional Development for Action and Change. LEARNing Landscapes Journal, Vol. 8 No. 2, 39-47. Canadá. Recuperado desde: https://www.learninglandscapes.ca/index. php/learnland/article/view/CommentaryInquiry-Based-Teacher-Learning-for-InclusivityProfessional-Development-for-Action-andChange

Zabalza, M. A. (2016). El Practicum y las prácticas externas en la formación universitaria. Revista Practicum, 1(1), 1-23.

Zeichner, K. (2016). Advancing social justice and democracy in teacher education: Teacher preparation 1.0, 2.0, and 3.0. Kappa Delta Pi Record, 52(4): Democracy and Social Justice, 150-155. Londres, Inglaterra y Gales. Recuperado desde: https://www.tandfonline. com/doi/full/10.1080/00228958.2016.1223986

\section{Anexo 1}

Cuestionario de evaluación de los estudiantes

\section{INSTITUTO SUPERIOR DE FORMACIÓN DOCENTE SALOMÉ UREÑA Recinto FÉLIX EVARISTO MEJÍA}

Instrumento de reflexión sobre la Participación en el Proyecto "Enseñar como Campeón". Técnica que será evaluada: "Sin Opción de Salida"

Estudiante:

Fecha: Grado: Profesor / tutor:

\begin{tabular}{|l|l|l|l|l|}
\hline $\begin{array}{l}\text { Marca cómo describes tu autopercepción respecto a las } \\
\text { siguientes afirmaciones. }\end{array}$ & Insatisfecho & Básico & Avanzado & Sobresaliente \\
\hline La participación en la clase permitió el aprendizaje. & & & & \\
\hline Fue fácil poner en práctica la técnica utilizada. & & & & \\
\hline Hubo respeto durante el proceso y el estudiantado. & & & \\
\hline La técnica ejecutada permitió el aprendizaje significativo. & & & & \\
\hline $\begin{array}{l}\text { Se pudo percibir la razón del aprendizaje y su impacto entre } \\
\text { compañeros. }\end{array}$ & & & & \\
\hline
\end{tabular}


Respondieron las preguntas siempre que les fue posible.

El docente se manejó, auxiliándose de otro alumno, para buscar la respuesta acertada.

El maestro regresó a buscar la respuesta en el alumno que no respondió.

Notas

\section{Anexo 2}

Cuestionario de autoevaluación sobre la satisfacción en la participación del programa de entrenamiento en técnicas de enseñanza

\section{INSTITUTO SUPERIOR DE FORMACIÓN DOCENTE SALOME UREÑA Autoevaluación sobre la satisfacción en la participación del programa de entrenamiento en técnicas de enseñanza}

Este cuestionario pretende evaluar tu satisfacción como participante en el programa de entrenamiento en técnicas didácticas vinculadas al proyecto "Enseñar como un campeón".

A continuación, encontrarás una lista de enunciados sobre tu percepción sobre las técnicas aprendidas y su posterior aplicación durante tu inserción en la escuela como estudiante en prácticas.

Solicitamos que respondas con absoluta sinceridad.

Nombre

Fecha_____/20__ Grado

Técnica

Sección I Sobre la valoración del programa

\begin{tabular}{|l|l|l|l|l|}
\hline & No aplica & A veces & Con frecuencia & Siempre \\
\hline Promueve el clima positivo & & & & \\
\hline Favorece la interacción entre docente y estudiante & & & & \\
\hline $\begin{array}{l}\text { Permite detectar necesidades individuales del estudiante: sus intereses, sus } \\
\text { motivaciones y capacidad para aprender }\end{array}$ & & & & \\
\hline Favorece el manejo de la conducta en el aula & & & & \\
\hline Permite una distribución del tiempo efectiva & & & & \\
\hline Promueve el aprendizaje significativo & & & & \\
\hline Mejora la calidad de la retroalimentación que se ofrece al estudiante & & & & \\
\hline Favorece la atención de los estudiantes & & & & \\
\hline
\end{tabular}

Sección II Sobre tu percepción de las técnicas

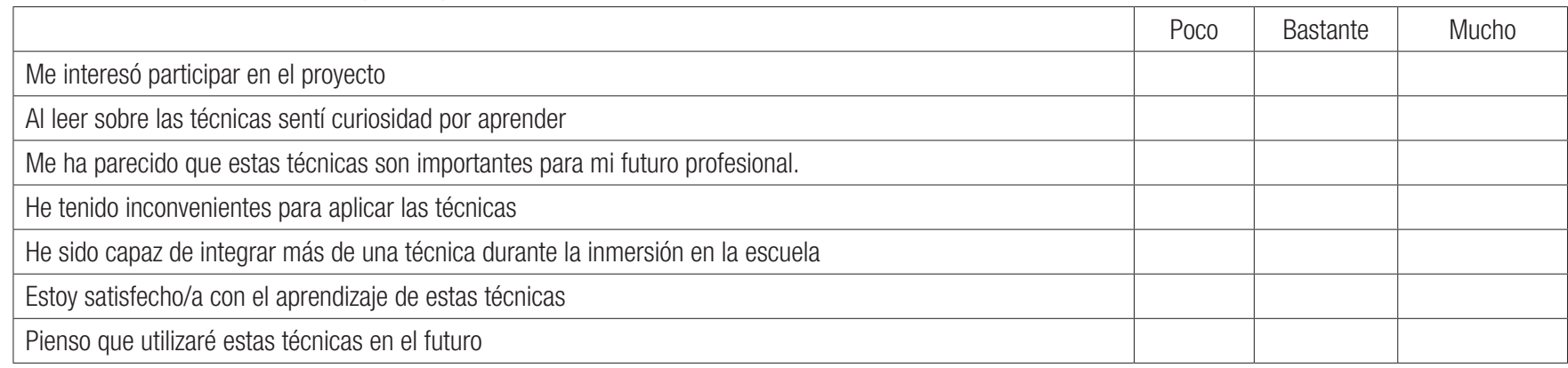

Sección III Sobre tu evaluación sobre el nivel de dominio de las técnicas

\begin{tabular}{|l|l|l|l|l|}
\hline & No aplica & Insuficiente & Básico & Avanzado \\
\hline Considero que mi nivel de dominio de esta técnica es & & & & \\
\hline
\end{tabular}

Observaciones: 